\title{
Partial Dynamical Symmetry at Critical-Points of Quantum Phase Transitions
}

\author{
A. Leviatan \\ Racah Institute of Physics, The Hebrew University, Jerusalem 91904, Israel
}

(Dated: November 12, 2017)

\begin{abstract}
We show that partial dynamical symmetries (PDS) can occur at critical-points of quantum phase transitions, in which case, underlying competing symmetries are conserved exactly by a subset of states, and mix strongly in other states. Several types of PDS are demonstrated with the example of critical-point Hamiltonians for first- and second-order transitions in the framework of the interacting boson model, whose dynamical symmetries correspond to different shape-phases in nuclei.
\end{abstract}

PACS numbers: 21.60.Fw, 21.10.Re

Symmetries play a profound role in determining the critical behaviour of dynamical systems. Their significance was recognized in Landau's classic theory of thermal phase transitions [1] and in the renormalization group of critical phenomena [2]. An equally-important role is played by symmetries in quantum phase transitions (QPT) or ground-state energy phase transitions [3], which occur at zero temperature as a function of a coupling constant. Such structural changes are currently of great interest in different branches of physics. QPT occur as a result of a competition between terms in the Hamiltonian with different symmetries which lead to considerable mixing in the eigenfunctions, especially at the critical-point where the structure changes most rapidly. In the present work we address the question whether there are any symmetries (or traces of) still present at the critical-points of QPT. We show that particular symmetry constructions, called partial dynamical symmetries, can survive at the critical-point in-spite of the strong mixing. The feasibility of such persisting symmetries gains support from the recently proposed [4] and empirically confirmed [5] analytic descriptions of critical-point nuclei, and the emergence of "quasi-dynamical symmetries" [6] in the vicinity of such critical-points.

A convenient framework to study symmetry-aspects of QPT are models where the Hamiltonian is expanded in elements of a Lie algebra $\left(G_{0}\right)$, called the spectrum generating algebra. A dynamical symmetry occurs if the Hamiltonian can be written in terms of the Casimir operators of a chain of nested algebras of $G_{0}$,

$$
G_{0} \supset G_{1} \supset \ldots \supset G_{n}
$$

terminating with an invariant algbera $G_{n}$. The following properties are then observed. (i) All states are solvable and analytic expressions are available for energies and other observables. (ii) All states are classified by quantum numbers, $\left|\alpha_{0}, \alpha_{1}, \ldots, \alpha_{n}\right\rangle$, which are the labels of the irreducible representations (irreps) of the algebras in the chain. (iii) The structure of wave functions is completely dictated by symmetry and is independent of the Hamiltonian's parameters. Partial dynamical symmetry (PDS) corresponds to a particular symmetry-breaking for which some (but not all) of the above mentioned virtues of a dynamical symmetry are retained. PDS of type I corresponds to a situation where some of the states have all the dynamical symmetry. In this case the properties (i)(iii) are fulfilled exactly, but by only a subset of states. PDS of type II corresponds to a situation for which all the states preserve part of the dynamical symmetry. In this case there are no analytic solutions, yet selected quantum numbers (of the conserved symmetries) are retained. This can occur, for example, when, the Hamiltonian preserves only selected symmetries $G_{i} \subset G_{n}$ in the chain (1), and only their irreps are unmixed. PDS of type III has a hybrid character, for which some of the states preserve part of the dynamical symmetry. PDS of various types have been shown to be relevant to nuclear and molecular spectroscopy [7, 8, 9, 10, 11, 12] and to mixed systems with coexisting regularity and chaos 13]. All examples of PDS encountered so far involved stable limits of structure. In the present work we show the relevance of the PDS notion to the more complicated case of a phase transition.

As a concrete example, we consider the interacting boson model (IBM) [14], widely used in the description of quadrupole collective states in nuclei, in terms of a system of $N$ monopole $(s)$ and quadrupole $(d)$ bosons, representing valence nucleon pairs. The spectrum generating algebra is $G_{0}=U(6)$ and the invariant algebra is $G_{n}=O(3)$. The three dynamical symmetry limits of the model and corresponding bases are

$$
\begin{array}{llll}
U(6) \supset U(5) \supset O(5) \supset O(3) & & \left|N, n_{d}, \tau, \tilde{\nu}, L\right\rangle \\
U(6) \supset S U(3) \supset O(3) & & |N,(\lambda, \mu), K, L\rangle \\
U(6) \supset O(6) \supset O(5) \supset O(3) & & |N, \sigma, \tau, \tilde{\nu}, L\rangle .
\end{array}
$$

The quantum numbers $N, n_{d},(\lambda, \mu), \sigma, \tau$ and $L$ label the relevant irreps of $U(6), U(5), S U(3), O(6), O(5)$ and $O(3)$ respectively. $\tilde{\nu}$ and $K$ are multiplicity-labels needed for complete classification of selected states in the reductions $O(5) \supset O(3)$ and $S U(3) \supset O(3)$ respectively. The analytic solutions of these dynamical symmetries resemble a spherical vibrator, axially-deformed rotor and deformed $\gamma$-soft rotor for the $U(5), S U(3)$ and $O(6)$ chains respectively. This identification is consistent with the geometric visualization of the model in terms of a potential surface 


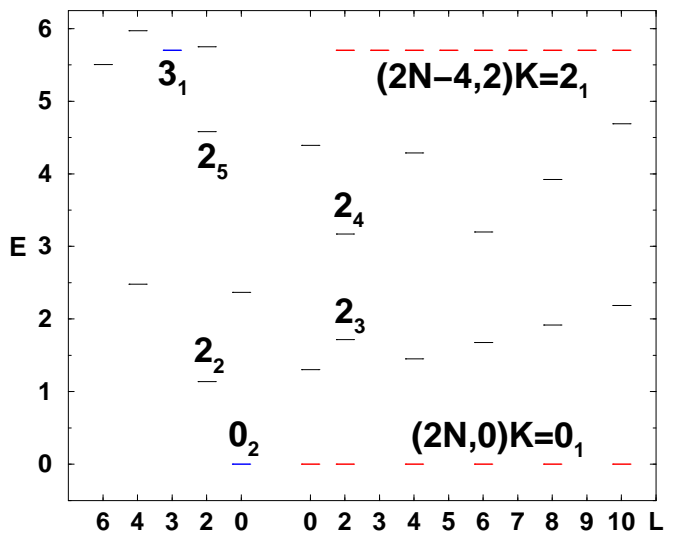

FIG. 1: (color online). Spectrum of $H\left(\beta_{0}=\sqrt{2}\right)$, Eq. (77), with $h_{2}=0.1$ and $N=10 . \quad L=0_{2}, 3_{1}$ are solvable $U(5)$ states of Eq. (9). $L\left(K=0_{1}\right)$ and $L\left(K=2_{1}\right)$ are, respectively, solvable $S U(3)$ states of Eq. (10a) and Eq. (10b) with $k=1$.

defined by the expectation value of the Hamiltonian in the coherent (intrinsic) state [15, 16]

$$
|\beta, \gamma ; N\rangle=(N !)^{-1 / 2}\left(b_{c}^{\dagger}\right)^{N}|0\rangle,
$$

where $b_{c}^{\dagger}=\left(1+\beta^{2}\right)^{-1 / 2}\left[\beta \cos \gamma d_{0}^{\dagger}+\beta \sin \gamma\left(d_{2}^{\dagger}+d_{-2}^{\dagger}\right) / \sqrt{2}+\right.$ $s^{\dagger}$. For the general IBM Hamiltonian with one- and twobody interactions, the potential surface reads

$$
E(\beta, \gamma)=E_{0}+N(N-1) \frac{\left[a \beta^{2}-b \beta^{3} \cos 3 \gamma+c \beta^{4}\right]}{\left(1+\beta^{2}\right)^{2}} .
$$

The coefficients $E_{0}, a, b, c$ involve particular linear combinations of the Hamiltonian's parameters [17]. The quadrupole shape parameters $(\beta, \gamma)$ at the global minimum of $E(\beta, \gamma)$ define the equilibrium shape for a given Hamiltonian. The shape can be spherical $(\beta=0)$ or deformed $(\beta>0)$ with $\gamma=0$ (prolate), $\gamma=\pi / 3$ (oblate), or $\gamma$-independent $(b=0)$.

Phase transitions can be studied by IBM Hamiltonians of the form, $H_{1}+g H_{2}$, involving terms from different dynamical symmetry chains [16]. The nature of the phase transition is governed by the topology of the corresponding surface (4), which serves as a Landau's potential with the equilibrium deformations as order parameters. The conditions on the surface at the critical-points of firstand second-order transitions are

$$
\begin{aligned}
& 1^{s t} \text { order } \quad b^{2}=4 a c, a>0, b \neq 0 \\
& 2^{\text {nd }} \text { order } \quad a=0, b=0, c>0 \text {. }
\end{aligned}
$$

The first-order critical-surface (5a) has degenerate spherical and deformed minima at $\beta=0$ and $\left(\beta=2 a /|b|, \gamma_{0}\right)$, with $\gamma_{0}=0(\pi / 3)$ for $b>0(b<0)$. The second-order critical-surface (5b) is independent of $\gamma$ and behaves as $\beta^{4}$ for small $\beta$. The conditions in Eq. (5) fix the critical value of the control parameter $\left(g=g_{c}\right)$ which, in turn,

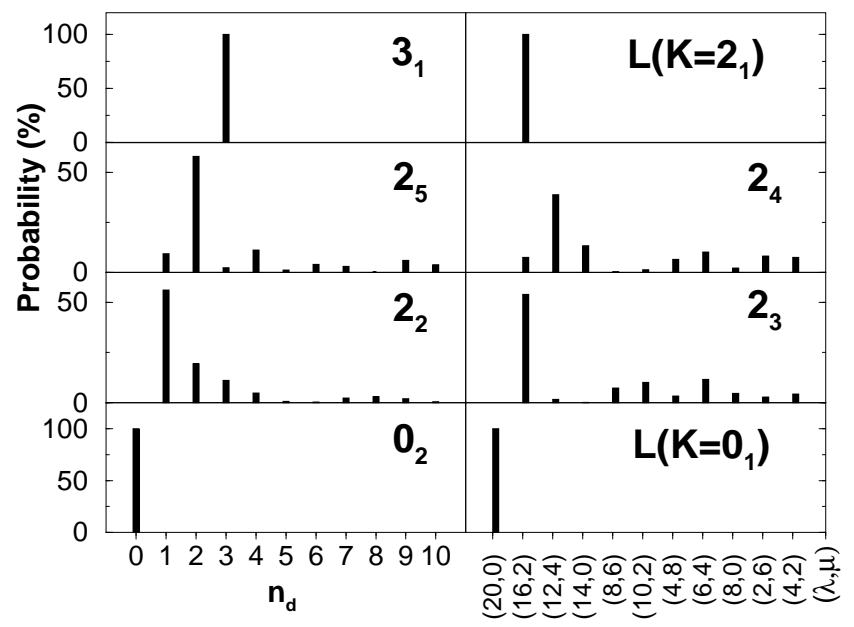

FIG. 2: $U(5)\left(n_{d}\right)$ and $S U(3)[(\lambda, \mu)]$ decomposition of selected spherical and deformed states in Fig. 1.

determines the critical-point Hamiltonian. IBM Hamiltonians of this type have been used extensively for studying shape-phase transitions in nuclei $[6,18,19,20,21]$. We now show that a large class of such Hamiltonians exhibit PDS.

The spherical to deformed $\gamma$-soft shape-phase transition is modeled in the IBM by the Hamiltonian

$$
\begin{aligned}
H & =\epsilon \hat{n}_{d}+A\left[d^{\dagger} \cdot d^{\dagger}-\left(s^{\dagger}\right)^{2}\right][\text { H.c. }] \\
\epsilon & =4(N-1) A
\end{aligned}
$$

where H.c. stands for Hermitian conjugate and the dot implies a scalar product. The $\hat{n}_{d}$-term is the $d$-boson number operator (eigenvalues $n_{d}$ ), which is the linear Casimir operator of $U(5)$. The $A$-term is related to the Casimir operator of $O(6)$ [14]. For the indicated ratio of coefficients, the above $H$ satisfies condition (5b), hence qualifies as a second-order critical Hamiltonian. The first (second) term in Eq. (6) has $O(6)[U(5)]$ selection rules $\Delta \sigma=0, \pm 2\left(\Delta n_{d}=0, \pm 2\right)$, and both terms are $O(5)$ scalars. Consequently, the eigenstates of $H$ have good $O(5)$ symmetry $(\tau)$, but are mixed strongly with respect to both $U(5)$ and $O(6)$ [19]. Since both $U(5)$ and $O(6)$ are broken while $O(5) \supset O(3)$ are preserved, by definition, the critical Hamiltonian has an $O(5)$ PDS of type II. In fact, since $O(5)$ is a good symmetry common to both chains (2a) and (2c), the $O(5)$ PDS is valid throughout the $U(5)-O(6)$ transition region.

A recent study of QPT within the IBM has shown that, apart from rotational terms which do not affect the potential surface of Eq. (4), the critical Hamiltonian for a spherical to prolate-deformed shape-phase transition can be transcribed in the form [21]

$$
H\left(\beta_{0}\right)=h_{2} P_{2}^{\dagger}\left(\beta_{0}\right) \cdot \tilde{P}_{2}\left(\beta_{0}\right),
$$

where $P_{2 \mu}^{\dagger}\left(\beta_{0}\right)=\beta_{0} s^{\dagger} d_{\mu}^{\dagger}+\sqrt{7 / 2}\left(d^{\dagger} d^{\dagger}\right)_{\mu}^{(2)}, \tilde{P}_{2 \mu}\left(\beta_{0}\right)=$ $(-1)^{\mu} P_{2,-\mu}\left(\beta_{0}\right)$ and $h_{2}, \beta_{0}>0$. The corresponding sur- 
face in Eq. (4) has coefficients $a=h_{2} \beta_{0}^{2}, b=2 h_{2} \beta_{0}, c=$ $h_{2}$, which satisfy condition (5a). This qualifies $H\left(\beta_{0}\right)$ as a first-order critical Hamiltonian whose potential accommodates two degenerate minima at $\beta=0$ and $(\beta, \gamma)=$ $\left(\beta_{0}, 0\right) . \quad H\left(\beta_{0}\right)$ is constructed to have the equilibrium intrinsic state, $\left|\beta=\beta_{0}, \gamma=0 ; N\right\rangle$, Eq. (3), as a zeroenergy eigenstate. Rotational-invariance ensures that states, $\left|\beta_{0} ; N, L\right\rangle$, of good $O(3)$ symmetry $L$ projected from this intrinsic state, remain zero-energy eigenstates. $H\left(\beta_{0}\right)$ then has a solvable deformed ground band,

$$
\left|\beta_{0} ; N, L\right\rangle \quad E=0 \quad(L=0,2,4, \ldots, 2 N) .
$$

It has also the following solvable spherical eigenstates

$$
\begin{aligned}
\left|N, n_{d}=\tau=L=0\right\rangle & E=0 \\
\left|N, n_{d}=\tau=L=3\right\rangle & E=3 h_{2}\left[\beta_{0}^{2}(N-3)+5\right] .
\end{aligned}
$$

As shown in Figs. 1-4, the remaining states in the spectrum of $H\left(\beta_{0}\right)$ are either predominantly spherical (with characteristic dominance of single $n_{d}$ components) or deformed states (with a broad $n_{d}$ distribution) arranged in several excited bands.

The critical Hamiltonian of Eq. (7) with $\beta_{0}=\sqrt{2}$ is a special case of a Hamiltonian shown in [7] to have $S U(3)$ PDS. This comes about because the sequence of states $|k\rangle \propto\left[P_{2,2}^{\dagger}(\sqrt{2})\right]^{k}|\beta=\sqrt{2}, \gamma=0 ; N-2 k\rangle$ are eigenstates of $H\left(\beta_{0}=\sqrt{2}\right)$. These are lowest-weight states in the $S U(3)$ irreps $(\lambda, \mu)=(2 N-4 k, 2 k)$ with $2 k \leq N$. In the nuclear physics terminology they are referred to as intrinsic states representing deformed ground $(k=0)$ and $\gamma^{k}$ bands, with angular momentum projection $(K=2 k)$ along the symmetry axis. Since $H\left(\beta_{0}=\sqrt{2}\right)$ is an $O(3)$ scalar, the states of good $L$ projected from $|k\rangle$ remain eigenstates with quantum numbers, $|N,(\lambda, \mu) K, L\rangle$, of

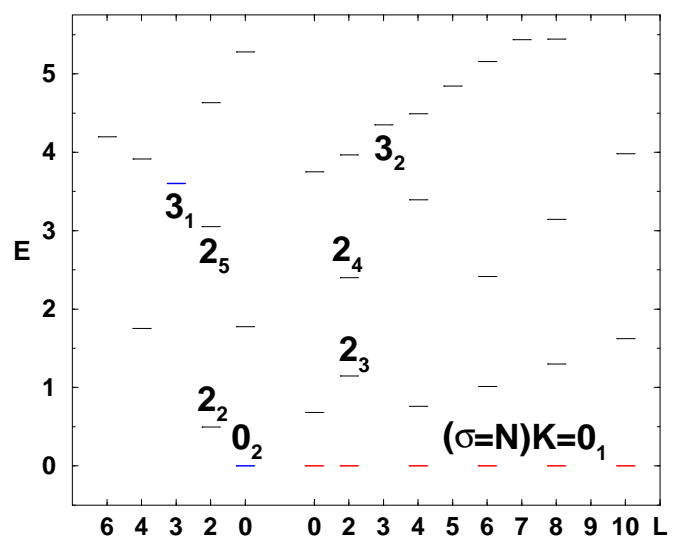

FIG. 3: (color online). Spectrum of $H\left(\beta_{0}=1\right)$, Eq. (7), with $h_{2}=0.1$ and $N=10 . L=0_{2}, 3_{1}$ are solvable $U(5)$ states of Eq. (9). $L\left(K=0_{1}\right)$ are solvable states of Eq. (11) with good $O(6)$ but broken $O(5)$ symmetry. the $S U(3)$ chain (2b), and form solvable bands,

$$
\begin{gathered}
|N,(2 N, 0) K=0, L\rangle E=0 \quad(L=0,2,4, \ldots, 2 N) \\
|N,(2 N-4 k, 2 k) K=2 k, L\rangle E=3 h_{2}[2 N+1-2 k] k \\
L=K, K+1, K+2, \ldots,(2 N-2 k) \quad k>0 . \quad
\end{gathered}
$$

In addition, $H\left(\beta_{0}=\sqrt{2}\right)$ has the spherical states of Eq. (9), with good $U(5)$ symmetry, as eigenstates. The remaining levels of $H\left(\beta_{0}=\sqrt{2}\right)$, shown in Fig. 1, are calculated numerically. Their wave functions are spread over many $U(5)$ and $S U(3)$ irreps, as is evident from Fig. 2. This situation, where some states are solvable with good $U(5)$ symmetry, some are solvable with good $S U(3)$ symmetry and all other states are mixed with respect to both $U(5)$ and $S U(3)$, defines a $U(5)$ PDS of type I coexisting with a $S U(3)$ PDS of type I.

The Hamiltonian of Eq. (7) with $\beta_{0}=1$ is a special case of a Hamiltonian shown in 9 ] to have $O(6)$ PDS. This comes about because the intrinsic state of Eq. (3) with $\left(\beta_{0}=1, \gamma=0\right)$ is a zero-energy eigenstate of $H\left(\beta_{0}=1\right)$ with good $O(6)$ symmetry $(\sigma=N)$. The $O(3)$-invariance of the Hamiltonian ensures that states of good $L$ projected from $\left|\beta_{0}=1, \gamma=0 ; N\right\rangle$ form a solvable ground band with good $O(6)$ character,

$$
|N, \sigma=N, L\rangle \quad E=0 \quad(L=0,2,4, \ldots, 2 N) .
$$

In addition, $H\left(\beta_{0}=1\right)$ has the spherical states of Eq. (9), with good $U(5)$ symmetry, as eigenstates. The remaining eigenstates in Fig. 3 are mixed with respect to both $U(5)$ and $O(6)$, as is evident from their decomposition shown in Fig. 4. Apart from the solvable $U(5)$ states of Eq. (9), all eigenstates of $H\left(\beta_{0}=1\right)$ are mixed with respect to $O(5)$ (including the solvable $O(6)$ states of Eq. (11), as shown in Fig. 5). It follows that the Hamiltonian has a subset of states with good $U(5)$ symmetry and a subset of states with good $O(6)$ but broken $O(5)$ symmetry, and

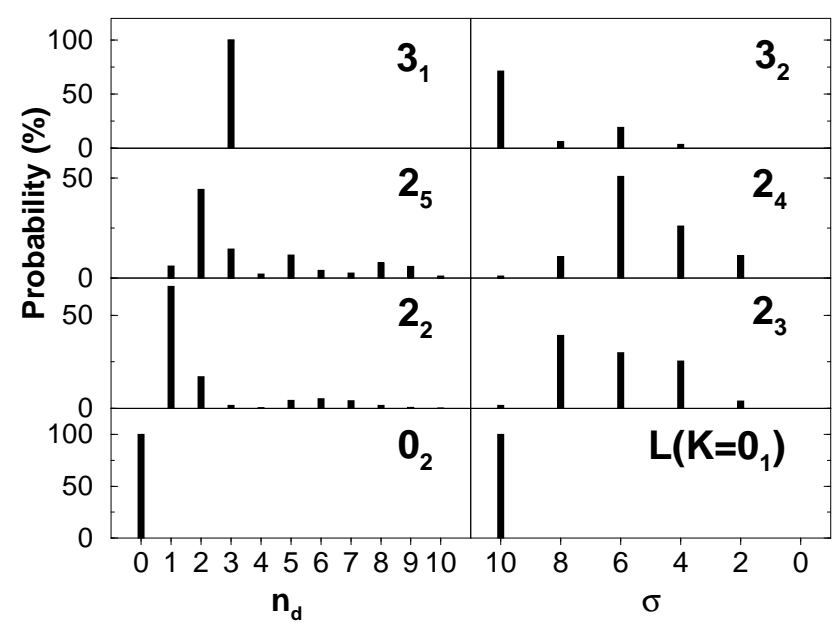

FIG. 4: $U(5)\left(n_{d}\right)$ and $O(6)(\sigma)$ decomposition of selected spherical and deformed states in Fig. 3. 


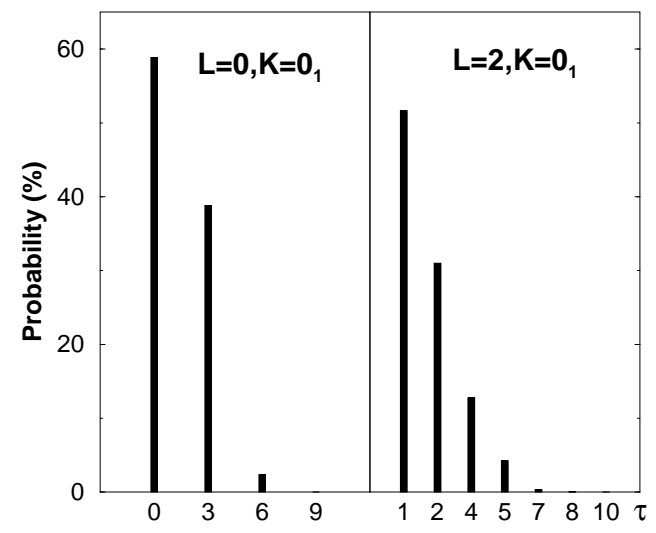

FIG. 5: $O(5)(\tau)$ decomposition of the $L=0,2$ states, Eq. (11), members of the ground band $\left(K=0_{1}\right)$ shown in Fig. 3. Both states have $O(6)$ symmetry $\sigma=N$.

all other states are mixed with respect to both $U(5)$ and $O(6)$. These are precisely the required features of $U(5)$ PDS of type I coexisting with $O(6)$ PDS of type III.

For arbitrary values of $\beta_{0}$, the spherical states with good $U(5)$ symmetry, Eq. (9), are still eigenstates of $H\left(\beta_{0}\right)$, Eq. (7), hence $U(5)$ PDS of type I is still valid. In general, the deformed states of Eq. (8), are not associated with any IBM dynamical symmetry but, nevertheless, are still solvable. This situation may be referred to as partial solvability. Since the wave functions of the solvable states are known, it is possible to obtain closed form expressions for related observables. For example, for the electromagnetic $E 2$ operator, $T(E 2)=d^{\dagger} s+s^{\dagger} \tilde{d}+\chi\left(d^{\dagger} \tilde{d}\right)^{(2)}$, the necessary matrix elements for transitions involving states in Eqs. (8)-(9) are $T_{1} \equiv\langle\beta ; N, L\|T(E 2)\| \beta ; N, L+2\rangle$ and $T_{2} \equiv\left\langle\beta ; N, L=2\|T(E 2)\| N, n_{d}=\tau=L=0\right\rangle$,

$$
\begin{aligned}
& T_{1}=C_{L} \frac{\beta\left[a_{1} \Gamma_{N-1}^{(L)}(\beta)+a_{2} \Gamma_{N-1}^{(L+2)}(\beta)\right]}{\left[\Gamma_{N}^{(L)}(\beta) \Gamma_{N}^{(L+2)}(\beta)\right]^{1 / 2}}, \\
& T_{2}=\beta N /\left[N ! \Gamma_{N}^{(2)}(\beta)\right]^{1 / 2}
\end{aligned}
$$

where $C_{L}=\sqrt{2 L+5}(L+2,0 ; 2,0 \mid L, 0)$ is proportional to a Clebsch Gordan coefficient, $a_{1}=1-\beta \bar{\chi} L /(2 L+3)$, $a_{2}=1-\beta \bar{\chi}(L+3) /(2 L+3)$ with $\bar{\chi}=\sqrt{2 / 7} \chi$, and $\Gamma_{N}^{(L)}(\beta)$ is a normalization factor given in [21].

As discussed, the spectrum of $H\left(\beta_{0}\right)$, Eq. (7), exhibits coexistence of spherical and deformed states, signaling a first-order transition. In particular, the spherical $L=0$ state, Eq. (9a), is exactly degenerate with the ground band, Eq. (8), and for $\beta_{0}=\sqrt{2}$ also the spherical $L=3$ state, Eq. (9b), is degenerate with the $S U(3) \gamma$-band, Eq. (10b) with $k=1$. Adding to the Hamiltonian the Casimir operator of $O(3)$, contributes an exact $L(L+1)$ splitting with no effect on wave functions. The remaining degeneracy of states with the same $L$, can be lifted by adding a small one-body term $\hat{n}_{d}$. With that, the spherical $U(5)$ states of Eq. (9) remain solvable eigenstates. However, the $\hat{n}_{d}$ term destroys the exact solvability and partial-symmetry of the deformed states, Eq. (8). The corresponding leading-order shifts can be estimated from $\left\langle\beta ; N, L\left|\hat{n}_{d}\right| \beta ; N, L\right\rangle=N-\Gamma_{N-1}^{(L)}(\beta) / \Gamma_{N}^{(L)}(\beta)$.

In summary, we have shown the relevance of the PDS notion to critical-points of QPT, with phases characterized by Lie-algebraic symmetries. In the example considered, second-order critical Hamiltonians mix incompatible symmetries but preserve a common lower symmetry, resulting in a single PDS with selected quantum numbers conserved. First-order critical Hamiltonians exhibit distinct subsets of solvable states with good symmetries, giving rise to a coexistence of different PDS. The ingredients of an algebraic description of QPT is a spectrum generating algebra and an associated geometric space, formulated in terms of coherent (intrinsic) states. The same ingredients are used in the construction of Hamiltonians with PDS. These, in accord with the present work, can be used as tools to explore the role of possibly partial symmetries in governing the critical behaviour of diverse dynamical systems undergoing QPT. This work was supported by the Israel Science Foundation.

[1] L.D. Landau and E.M. Lifshitz, "Statistical Physics", 3rd ed. Pergamon, Oxford, 1980.

[2] K.G. Wilson and J. Kogut, Phys. Rep. 12, 75 (1974).

[3] R. Gilmore, J. Math. Phys. 20, 891 (1979).

[4] F. Iachello, Phys. Rev. Lett. 85, 3580 (2000); 87, 052502 (2001).

[5] R.F. Casten and N.V. Zamfir, Phys. Rev. Lett. 85, 3584 (2000); 87, 052503 (2001).

[6] D.J. Rowe, Nucl. Phys. A 745, 47 (2004); P.S. Turner and D.J. Rowe, ibid.756, 333 (2005); G. Rosensteel and D.J. Rowe, ibid. 759, 92 (2005).

[7] A. Leviatan, Phys. Rev. Lett. 77, 818 (1996).

[8] P. Van Isacker, Phys. Rev. Lett. 83, 4269 (1999).

[9] A. Leviatan and P. Van Isacker, Phys. Rev. Lett. 89, 222501 (2002), and references therein.

[10] J. Escher and A. Leviatan, Phys. Rev. Lett. 84, 1866 (2000); Phys. Rev. C 65, 054309 (2002).

[11] G. Rosensteel and D.J. Rowe, Phys. Rev. C 67, 014303 (2003).

[12] J.L. Ping and J.Q. Chen, Ann. Phys. 255, 75 (1997).

[13] N. Whelan, Y. Alhassid and A. Leviatan, Phys. Rev. Lett. 71, 2208 (1993); A. Leviatan and N.D. Whelan, Phys. Rev. Lett. 77, 5202 (1996).

[14] F. Iachello and A. Arima, The Interacting Boson Model, Cambridge Univ. Press, Cambridge 1987.

[15] J. N. Ginocchio and M. W. Kirson, Phys. Rev. Lett. 44, 1744 (1980).

[16] A. E. L. Dieperink, O. Scholten and F. Iachello, Phys. Rev. Lett. 44, 1747 (1980).

[17] A. Leviatan, Ann. Phys. (NY) 179, 201 (1987).

[18] F. Iachello and N.V. Zamfir, Phys. Rev. Lett. 92, 212501 (2004), and references therein.

[19] A. Leviatan and J. N. Ginocchio, Phys. Rev. Lett. 90, 212501 (2003).

[20] A. Leviatan, Phys. Rev. C 72, 031305(R) (2005).

[21] A. Leviatan, Phys. Rev. C 74, 051301(R) (2006). 